\section{Post-Mortem Evaluation of Amyloid-Dopamine Terminal Positron Emission Tomography Dementia Classifications}

\author{
Roger L. Albin, MD, 1,2,3,4 \\ Amanda Fisher-Hubbard, $\mathrm{MD}^{5}$ \\ Krithika Shanmugasundaram, BA, ${ }^{2}$ \\ Robert A. Koeppe, $\mathrm{PhD}^{6}{ }^{6}$ \\ James F. Burke, MD, MS, ${ }^{1,2,3}$ \\ Sandra Camelo-Piragua, MD, ${ }^{5}$ \\ Andrew P. Lieberman, MD, PhD, ${ }^{5}$ \\ Bruno Giordani, $\mathrm{PhD}^{7}$ and \\ Kirk A. Frey, MD, PhD ${ }^{2,6}$
}

\begin{abstract}
Clinical classification of early dementia and mild cognitive impairment $(\mathrm{MCl})$ is imprecise. We reported previously that molecular imaging classification of early dementia and $\mathrm{MCl}$ with dual amyloid and dopamine terminal positron emission tomography differs significantly from expert clinical classification. We now report pathological diagnoses in a substantial subset of our previously imaged subjects. Among 36 subjects coming to autopsy, imaging classifications and pathological diagnosis were concordant in 33 cases $(\kappa=0.85)$. This approach enhanced specificity of Alzheimer's disease diagnosis. The strong concordance of imaging-based classifications and pathological diagnoses suggests that this imaging approach will be useful in establishing more accurate and convenient classification biomarkers for dementia research.
\end{abstract}

ANN NEUROL 2015;78:824-830

C inical classification of dementias, particularly in early disease phases, is imprecise. ${ }^{1}$ There are three common neurodegenerative dementias; Alzheimer disease (AD), Lewy body dementia (LBD), and Frontotemporal dementias (FTDs). Even expert clinical characterization does relatively poorly in differentiating $\mathrm{AD}$ from FTDs. ${ }^{2}$ Clinical criteria for LBD possess good specificity, but relatively poor sensitivity. ${ }^{3}$ Mild cognitive impairment (MCI), a common precursor of dementia, is a heterogeneous category associated with all major neurodegenerative pathologies and vascular etiologies. Imprecise classification of MCI and early dementia subjects is an obstacle to clinical research owing to the fact that heterogeneous study populations dilute power to detect effects of trial interventions or associations with potential biomarkers. The emergence of positron emission tomography (PET) ligands identifying specific pathological features of neurodegenerative disorders raises the possibility of minimally invasive characterization of MCI and early dementia subjects. We previously reported results of combined amyloid ( $\left.\left[{ }^{11} \mathrm{C}\right] \mathrm{PIB}\right)$ and dopamine terminal $\left(\left[{ }^{11} \mathrm{C}\right] \mathrm{DTBZ}\right)$ PET imaging in $102 \mathrm{MCI}$ and early dementia subjects, demonstrating only moderate concordance $(\kappa=0.41)$ between imaging-based and expert clinical consensus classifications. ${ }^{4,5}$ Our previous results raise the possibility that this imaging-based approach to classification more faithfully reflects underlying pathologies than clinical characterization. We now report neuropathological follow-up of a substantial fraction of our study subjects.

\section{Subjects and Methods}

Study participants were individuals with MCI or relatively mild dementia (Mini-Mental State Examination [MMSE] > 17) as described previously and enrolled in our previous imaging study from 2005 to $2009.4,5$ The purpose of the previous study was to compare amyloid-dopamine terminal PET-based classification of early cognitive impairment subjects with expert clinical classification. Subjects with primary features of cognitive impairment were recruited from the University of Michigan Cognitive Disorders Clinic. Patients with primary neurological presentations involving noncognitive domains (ataxia, parkinsonism, and so on) were excluded. Inclusion-exclusion criteria are described in previous publications; patients with possible vascular dementia (modified Hachinski score $>4$ or meeting NINDS-AIREN criteria or large infarcts on structural imaging) were excluded. ${ }^{4}$ Clinical classifications were established by expert consensus conference based on clinical and

From the ${ }^{1}$ Neurology Service \& GRECC, VAAAHS, Ann Arbor, MI ${ }^{2}$ Department of Neurology, University of Michigan, Ann Arbor, Ml; ${ }^{3}$ Michigan Alzheimer's Disease Center, Ann Arbor, Ml; ${ }^{4}$ University of Michigan Morris K. Udall Center of Excellent for Parkinson's Disease Research, Ann Arbor, Ml; ${ }^{5}$ Department of Pathology, University of Michigan, Ann Arbor, MI; ${ }^{6}$ Division of Nuclear Medicine and Molecular Imaging, Department of Radiology, University of Michigan, Ann Arbor, Ml; and ${ }^{7}$ Department of Psychiatry, University of Michigan, Ann Arbor, MI

Address correspondence to Dr Roger L. Albin, 5023 BSRB, 109 Zina Pitcher Place, Ann Arbor, MI, 48109-2200. E-mail: ralbin@umich.edu

Additional supporting information can be found in the online version of this article.

Received Feb 6, 2015, and in revised form Jul 13, 2015. Accepted for publication Jul 13, 2015.

View this article online at wileyonlinelibrary.com. DOI: 10.1002/ana. 24481 
neuropsychological data accumulated at the time of visits for imaging, as described previously. ${ }^{4}$ Enrollees agreed to follow-up autopsy. To date, 41 study participants died and autopsies were completed on 36. Autopsy results of 1 subject were reported previously. ${ }^{6}$ All autopsies were performed at the University of Michigan Health System. Neuropathology was assessed by standard methods and using standard diagnostic criteria. ${ }^{7-11}$ The examining neuropathologists (A.F.-H., A.P.L., and S.C.-P.) were blind to results of imaging studies. Thal scores of amyloid plaque density were compiled for three neocortical regions; mid-frontal (Brodmann's areas [BA] 10 and 46), parietal (BA 7 and 39), and primary occipital (BA 17). Plaques were identified with $\mathrm{A} \beta$ immunohistochemistry (6F/3D; 1:50; Leica Biosystems, Nussloch, Germany). Thal scoring was available for all subjects. Regional $\left[{ }^{11} \mathrm{C}\right] \mathrm{PiB}$ binding was quantified as distribution volume ratios (DVRs) with the cerebellar gray matter as the reference region. Image-based classifications established in our previous studies were used for categorical comparison with pathological diagnoses. ${ }^{4,5}$ Standardized DVR image data sets were classified qualitatively by an expert interpreter (K.A.F.) familiar with the normal and pathological distributions of these tracers and blind to all clinical and routine structural imaging data, as described previously. ${ }^{4}$ In our previous study, use of parametric regional DVR thresholds for classification did not alter results. ${ }^{4}$ The unweighted Cohen's kappa statistic was used to estimate concordance between imaging based and pathological classifications. Spearman's rank-order correlation was used to compare amyloid burden assessed pathologically with the $\left[{ }^{11} \mathrm{C}\right] \mathrm{PiB}$ DVR estimates of regional amyloid burden. Sixteen subjects also underwent $\left[{ }^{18} \mathrm{~F}\right]$ fluorodeoxyglucose PET (FDGPET) at the same time they underwent DTBZ-PiB imaging. These studies were interpreted by the same expert interpreter (K.A.F.) blind to the clinical histories, and structural and PET imaging data.

\section{Results}

There was overall excellent concordance of imaging based classifications with neuropathological diagnoses $(\kappa=0.85 ; 95 \%$ confidence interval $=0.69-1.0$; Table 1 ; details of pathological results in Supplementary Table). Regional amyloid DVRs correlated well with neuropathological scoring of amyloid burden in the selected neocortical regions (Fig). For mid-frontal cortex, rho $=0.72$; for parietal cortex, rho $=0.79$; for primary occipital cortex, rho $=0.64$ (all $p<0.05)$. There were 3 cases with discordant imaging-pathological classifications. One subject had a clinical diagnosis and imaging classification of LBD, but a pathological diagnosis of AD. Alphasynuclein immunoreactive Lewy bodies were found in midbrain neurons in this subject, suggesting the presence of mixed AD-LBD pathology. The second discordant subject had marked frontal and temporal atrophy secondary to multiple small infarctions and imaging classification as FTD. The final discordant case was classified by

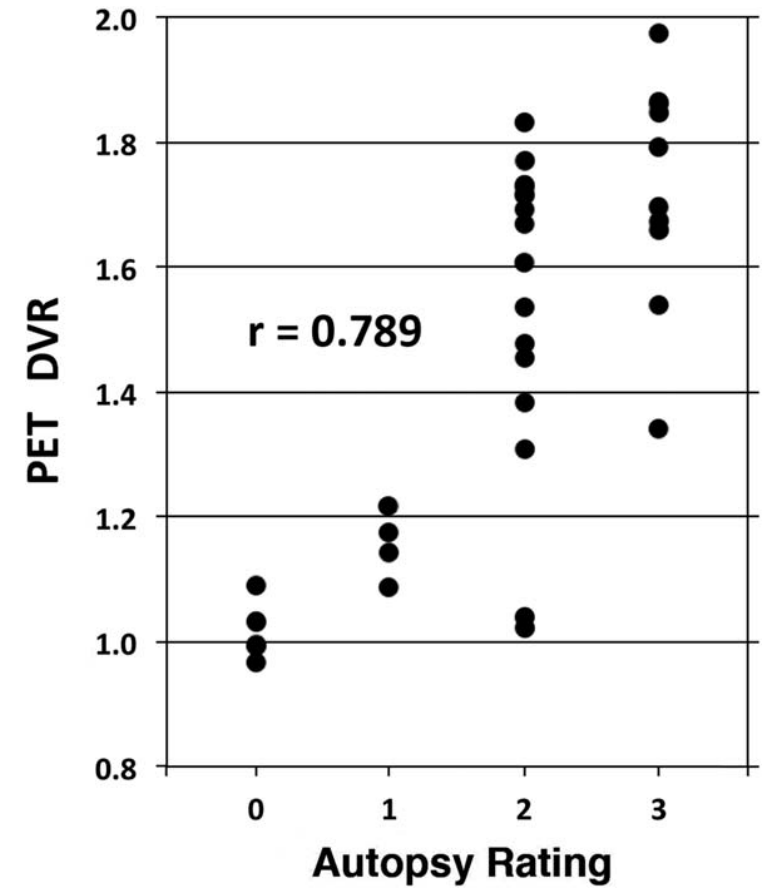

FIGURE 1: Scatter plot of parietal cortex Thal scores (autopsy rating) versus $\left[{ }^{11} \mathrm{C}\right] \mathrm{PiB}$ DVRs. $\mathrm{PET}=$ positron emission tomography; $\mathrm{DVR}=$ distribution volume ratio; $\left[{ }^{11} \mathrm{C}\right] \mathrm{PiB}=$ Pittsburgh $\mathrm{B} ; \mathrm{r}=$ Spearman's rho.

imaging as LBD, but remarkable only for the presence of transactive response DNA binding protein $43 \mathrm{kDa}$ (TDP-43)-immunoreactive neurites in the frontal cortex and hippocampal formation. This was an unusual case in that there was marked unilateral striatal loss of $\left[{ }^{11} \mathrm{C}\right] \mathrm{DTBZ}$ binding. Three cases were assessed pathologically as meeting criteria for both $\mathrm{AD}$ and LBD. These individuals had imaging classifications as LBD with amyloid deposition and are assessed as concordant classifications. There was excellent concordance between imaging assessments of increased amyloid burden and pathological results; all subjects found to have moderate-to-high amyloid plaque burden at autopsy were classified as amyloid positive in imaging classifications.

We performed a more limited comparison of combined amyloid and dopamine terminal imaging classifications, neuropathological diagnoses, and FDG-PET classifications. Approximately $30 \%$ of the FDG-PET classifications differed from final neuropathological diagnoses (Table 2). There were 3 cases where the FDG-PET classification was FTD with pathological diagnoses of AD and 2 cases where the FDG-PET classification was $\mathrm{AD}$ with pathological diagnoses of LDB. In all cases with discrepant FDG-PET classifications and neuropathological diagnoses, combined amyloid and dopamine terminal PET imaging correctly identified the pathological diagnosis (Table 2). 


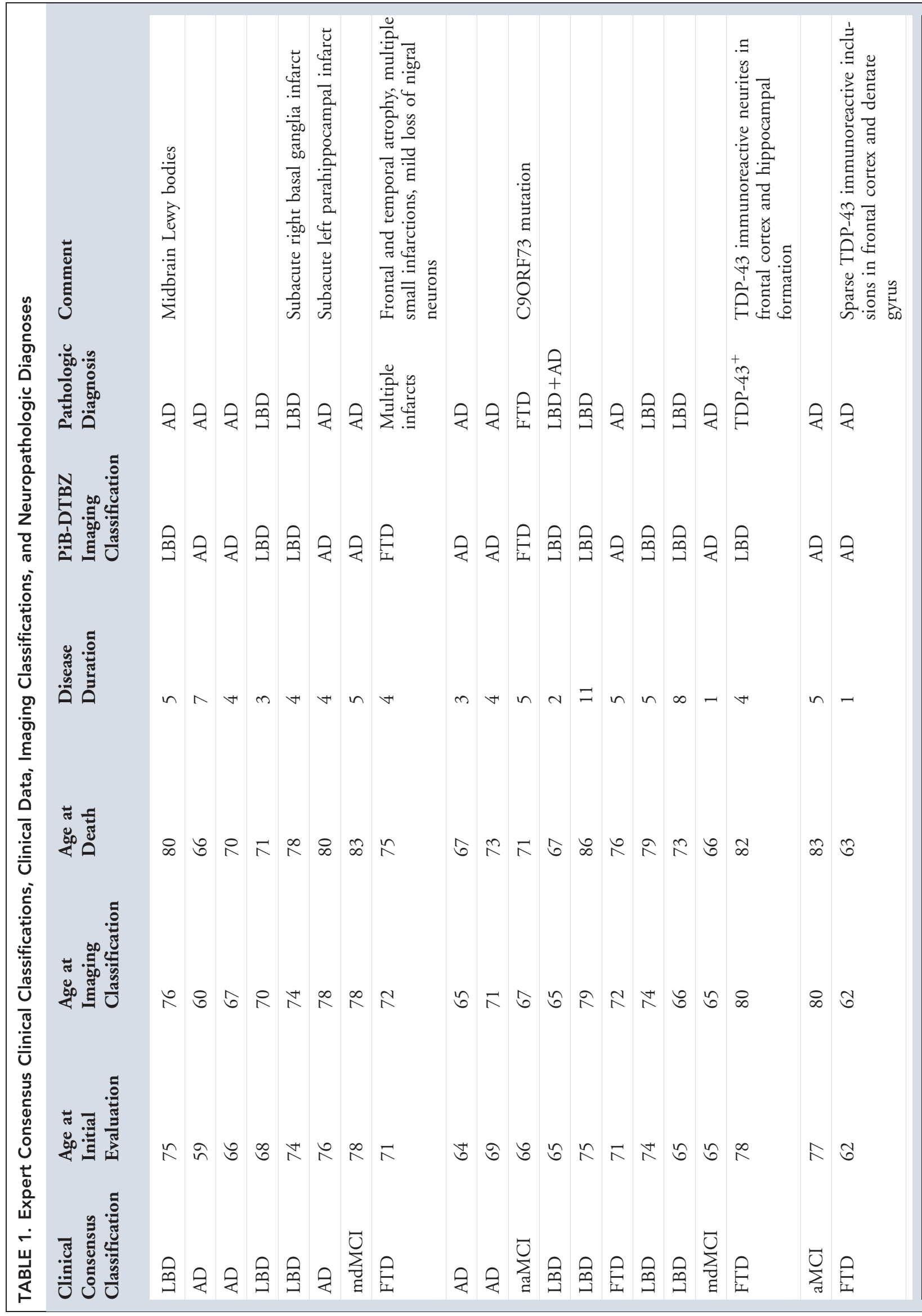




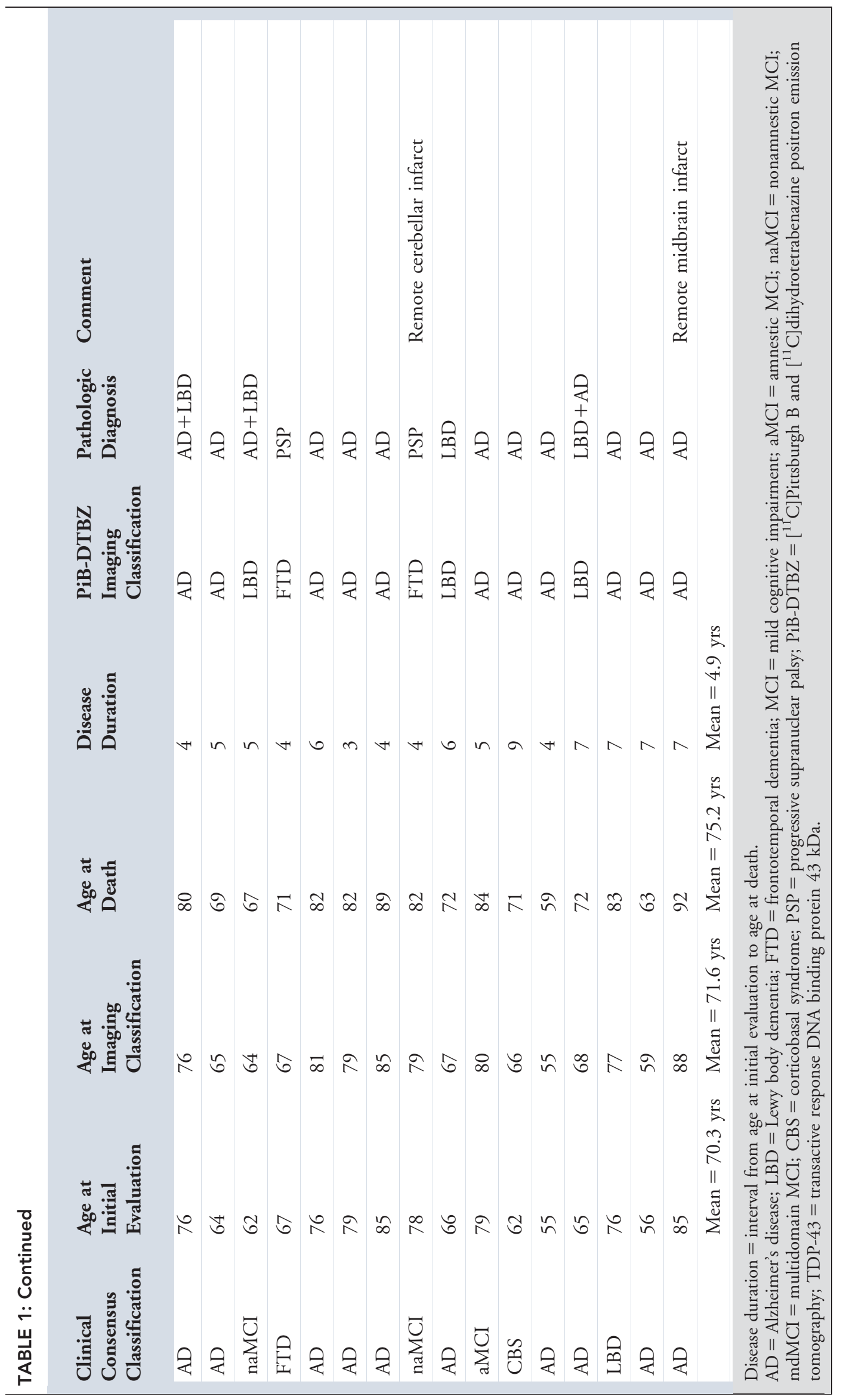




\section{Discussion}

Our results indicate that classifications based on combined amyloid and dopamine terminal PET imaging correlate well with neuropathological diagnostic classifications. Of 36 subjects studied, there were 3 cases (8.3\%) where imaging based and pathological classifications differed. In our previous studies, in contrast, $\sim 35 \%$ of participants had discordant expert clinical consensus and imaging diagnostic classifications. ${ }^{4,5}$ One discordant case was classified as LBD on the basis of significantly reduced striatal $\left[{ }^{11} \mathrm{C}\right] \mathrm{DTBZ}$ binding. Though not meeting pathological criteria for LBD, this subject had nigral Lewy bodies, suggesting mixed pathology.

Our results are consistent with other recent studies. In trials of antiamyloid therapy of clinically classified early $\mathrm{AD}$ subjects where participants underwent amyloid imaging, $\sim 15 \%$ of enrolled subjects had negative amyloid imaging, excluding AD. ${ }^{12-14}$ These results likely underestimate diagnostic misclassifications given that $\sim 50 \%$ of LBD cases exhibit significant amyloid burden, likely leading to misclassification of some LBD subjects as $\mathrm{AD} .{ }^{15} \mathrm{~A}$ clinicopathological study using the National Alzheimer's Coordinating Center (NACC) data set found that $\sim 15 \%$ of classified clinically $\mathrm{AD}$ subjects failed pathological criteria for $\mathrm{AD} .{ }^{16}$ In our previous studies, a major cause of discrepant clinical and imaging classifications were subjects classified clinically as FTD, but with positive amyloid imaging results suggesting $\mathrm{AD} .{ }^{4}$ Our results comparing amyloid/dopamine terminal imaging and clinical diagnostic classifications are similar to those reported by Beach et al. using the NACC data set to compare clinical and neuropathological diagnoses. ${ }^{17}$

Our limited evaluation of FDG-PET classifications suggests that this method is less precise than combined amyloid and dopamine terminal PET imaging. These types of FDG-PET misclassifications are well described in previous literature. Disproportionate frontal amyloid deposition may give rise to frontal predominant hypometabolism. ${ }^{18}$ The canonical pattern of cerebral metabolic deficits in LDB is the pattern of temporoparietal and frontal deficits found in $\mathrm{AD}$ plus occipital hypometabolism, but the distinguishing occipital metabolic deficits are absent is a significant fraction of patients. ${ }^{19}$

Amyloid imaging is accepted as a useful biomarker of fibrillar amyloid deposition. The high prevalence of amyloidopathy in LBD, however, indicates that increased amyloid burden is not a unique AD biomarker. Combining amyloid imaging with a dopamine terminal marker enhances accuracy. Our study, and this approach in general, has some limitations. Our number of autopsied subjects is relatively small. Because amyloid imaging is rela- tively sensitive for detecting $\mathrm{AD}$, and dopamine terminal imaging allows exclusion of LBD, this method is arguably best at improving identification of $\mathrm{AD}$. Two of the imaging misclassifications assessed subjects as LBD were found at autopsy to have another diagnosis. This result and the existence of nigrostriatal pathology in FTD and related syndromes indicate that dopamine terminal imaging possesses good sensitivity, but less specificity, for detection of LBD. In amyloid-negative individuals, substantial nigrostriatal terminal loss could indicate either LBD or FTD, given that some FTD patients develop parkinsonism with nigrostriatal degeneration, particularly those with MAPT or GRN mutations, decreasing the specificity of this approach of accurate classification of LBD. ${ }^{20}$ Identification of FTD is most problematic given that our classification of FTD is based on negative imaging results- the absence of pathological amyloid or dopamine terminal imaging changes. This may be misleading because there are multiple potential causes of cognitive impairment without amyloid or nigrostriatal pathology, for example, our case where the neuropathological evaluation revealed multiple small infarcts instead of neurodegeneration. Positive imaging markers for tau deposition and other FTD-associated pathologies would be useful additions to this imaging approach. ${ }^{21}$

Our results point to another problem secondary to use of the trinary classification scheme. This conventional approach is artificial in that mixed pathologies are common, though the presence of other pathologies does not confound amyloid ligand binding. ${ }^{22}$ Mixed pathologies are observed in our data set with 3 subjects with both $\mathrm{AD}$ and $\mathrm{LBD}$, and the discordant subject who met neuropathological criteria for $\mathrm{AD}$ and had midbrain Lewy bodies. Identification of individuals with both $\mathrm{AD}$ and LBD is particularly difficult, both with our dual tracer approach and with conventional clinical classifications. This is an area where addition of a tau tracer may be valuable.

Our study has significant advantages. Our subjects were enrolled during relatively early disease stages, either MCI status or relatively mild dementia (MMSE $>17$ ). Previous studies correlating amyloid imaging results with neuropathology enrolled individuals with advanced dementia. ${ }^{23-26}$ Our study population is more typical of clinical research studies and offers reassurance that previous imaging-pathological correlation studies of amyloid imaging are relevant to earlier phases of neurodegeneration. Our study design may underestimate the utility of this multitracer approach. Imaging classifications were made in the absence of clinical and structural (computed tomography or magnetic resonance imaging) imaging information. Conversely, our clinical classifications were 
TABLE 2. Expert consensus clinical classifications, PiB-DTBZ imaging classifications, neuropathological diagnoses, and FDG-PET classifications

\begin{tabular}{|c|c|c|c|c|c|c|c|}
\hline $\begin{array}{l}\text { Clinical } \\
\text { Consensus } \\
\text { Classification }\end{array}$ & $\begin{array}{l}\text { Age at } \\
\text { Initial } \\
\text { Evaluation }\end{array}$ & $\begin{array}{l}\text { Age at } \\
\text { Imaging } \\
\text { Classification }\end{array}$ & $\begin{array}{l}\text { Age at } \\
\text { Death }\end{array}$ & $\begin{array}{l}\text { Disease } \\
\text { Duration }\end{array}$ & $\begin{array}{l}\text { PiB-DTBZ } \\
\text { Imaging } \\
\text { Classification }\end{array}$ & $\begin{array}{l}\text { Pathologic } \\
\text { Diagnosis }\end{array}$ & $\begin{array}{l}\text { FDG-PET } \\
\text { Classification }\end{array}$ \\
\hline $\mathrm{AD}$ & 59 & 60 & 66 & 7 & $\mathrm{AD}$ & $\mathrm{AD}$ & $\mathrm{AD}$ \\
\hline LBD & 68 & 70 & 71 & 3 & LBD & LBD & $\mathrm{AD}$ \\
\hline $\mathrm{AD}$ & 64 & 65 & 67 & 3 & $\mathrm{AD}$ & $\mathrm{AD}$ & FTD \\
\hline $\mathrm{AD}$ & 69 & 71 & 73 & 4 & $\mathrm{AD}$ & $\mathrm{AD}$ & $\mathrm{AD}$ \\
\hline LBD & 75 & 79 & 86 & 11 & LBD & LBD & LBD \\
\hline LBD & 74 & 74 & 79 & 5 & LBD & LBD & LBD \\
\hline LBD & 65 & 66 & 73 & 8 & LBD & LBD & $\mathrm{AD} / \mathrm{LBD}$ \\
\hline mdMCI & 65 & 65 & 66 & 1 & $\mathrm{AD}$ & $\mathrm{AD}$ & $\mathrm{AD}$ \\
\hline aMCI & 77 & 80 & 83 & 5 & $\mathrm{AD}$ & $\mathrm{AD}$ & FTD \\
\hline $\mathrm{AD}$ & 76 & 81 & 82 & 6 & $\mathrm{AD}$ & $\mathrm{AD}$ & $\mathrm{AD}$ \\
\hline $\mathrm{AD}$ & 85 & 85 & 89 & 4 & $\mathrm{AD}$ & $\mathrm{AD}$ & FTD \\
\hline $\mathrm{AD}$ & 66 & 67 & 72 & 6 & LBD & LBD & $\mathrm{AD}$ \\
\hline aMCI & 79 & 80 & 84 & 5 & $\mathrm{AD}$ & $\mathrm{AD}$ & $\mathrm{AD}$ \\
\hline $\mathrm{AD}$ & 65 & 68 & 72 & 7 & LBD & $\mathrm{LBD}+\mathrm{AD}$ & LBD \\
\hline LBD & 76 & 77 & 83 & 7 & $\mathrm{AD}$ & $\mathrm{AD}$ & $\mathrm{AD}$ \\
\hline $\mathrm{AD}$ & 56 & 59 & 63 & 7 & $\mathrm{AD}$ & $\mathrm{AD}$ & $\mathrm{AD}$ \\
\hline \multicolumn{8}{|c|}{$\begin{array}{l}\text { Disease duration = interval from age at initial evaluation to age at death. } \\
\mathrm{AD}=\text { Alzheimer's disease; } \mathrm{LBD}=\text { Lewy body dementia; } \mathrm{FTD}=\text { frontotemporal dementia; } \mathrm{MCI}=\text { mild cognitive impairment; } \\
\mathrm{aMCI}=\text { amnestic } \mathrm{MCI} \text {; } \mathrm{mCCI}=\text { multidomain } \mathrm{MCI} \text { PiB-DTBZ }=\left[{ }^{11} \mathrm{C}\right] \mathrm{Pittsburgh} \mathrm{B} \text { and }\left[{ }^{11} \mathrm{C}\right] \text { dihydrotetrabenazine positron } \\
\text { emission tomography; FDG-PET }=\left[{ }^{18} \mathrm{~F}\right] \text { fluorodeoxyglucose/positron emission tomography. FDG-PET classification criteria: } \mathrm{AD} \text { : } \\
\text { temporoparietal and posterior cingulate hypometabolism; LDB: temporoparietal and posterior cingulate hypometabolism plus occi- } \\
\text { pital hypometabolism; FTD: frontal, anterior temporal, and anterior cingulate hypometabolism. }\end{array}$} \\
\hline
\end{tabular}

made without the PET results. Use of this multitracer PET approach in conjunction with clinical and structural imaging data would likely enhance accuracy of classifications. These methods may provide additional useful data. We showed previously that regional cerebral blood flow data derived from $\left[{ }^{11} \mathrm{C}\right] \mathrm{DTBZ}$ PET closely mimics the patterns of regional cerebral metabolism visualized with $\left[{ }^{18} \mathrm{~F}\right]$ FDG-PET imaging. ${ }^{27,28}$ Analysis of $\left[{ }^{11} \mathrm{C}\right] \mathrm{DTBZ}-$ based regional perfusion data would add a functional dimension to analysis and might further enhance classifications. Individuals, for example, with abnormal striatal $\left[{ }^{11} \mathrm{C}\right] \mathrm{DTBZ}$ ligand binding could have either LBD or FTD. These syndromes exhibit distinctive regional cerebral metabolic-perfusion deficit patterns, which could be helpful in classifying LBD and FTD subjects more accurately.

We do not suggest that this approach to classification would be broadly useful in clinical practice. It is more plausible that this approach, or approaches using similar tracers or incorporating additional tracers, such as a tau ligand, will be useful in clinical research. These methods may allow purer subject samples or better subject stratification, particularly for selection of AD subjects, for clinical research studies. This approach may be useful in establishing the utility of more accessible classification biomarkers. Rather than waiting years for autopsy results, this dual tracer approach or similar methods could be used as surrogates to validate more convenient classification biomarkers.

\section{Acknowledgment}

This work was supported by AG08671, NS15655, R56 NS082941, P50 NS091856, and a gift from an anonymous donor.

We thank the families of our research participants for their commitment to research. We thank the two anonymous reviewers for helpful criticisms. 


\section{Authorship}

R.L.A., K.A.F., and J.F.B. were responsible for study concept and design. R.L.A., K.A.F., R.A.K., J.F.B., B.G., A.F.-H., A.P.L., S.C.-P., and K.S. were responsible for data acquisition and analysis. R.L.A., K.A.F., and R.A.K. were responsible for drafting text and figures.

\section{Potential Conflicts of Interest}

Nothing to report.

\section{References}

1. Knopman DS, DeKosky ST, Cummings JL, et al. Practice parameter: diagnosis of dementia (an evidence-based review). Report of the quality standards subcommittee of the American Academy of Neurology. Neurology 2001;56:1143-1153.

2. Mendez MF, Shapira JS, McMurtray A, Licht E, Miller BL. Accuracy of the clinical evaluation for frontotemporal dementia. Arch Neurol 2007;64:830-835

3. McKeith IG, Ballard CG, Perry RH, et al. Prospective validation of consensus criteria for the diagnosis of dementia with Lewy bodies. Neurology 2000;54:1050-1058.

4. Burke JF, Albin RL, Koeppe RA, et al. Assessment of mild dementia with amyloid and dopamine terminal positron emission tomography. Brain 2011;134:1647-1657.

5. Albin RL, Burke JF, Koeppe RA, et al. Assessing mild cognitive impairment with amyloid and dopamine terminal molecular imaging. J Nucl Med 2013;54:887-893.

6. Meisler MH, Grant AE, Jones JM, et al. C9ORF72 expansion in a family with bipolar disorder. Bipolar Disord 2013;15:326-332.

7. Hyman BT, Phelps $\mathrm{CH}$, Beach TG, et al. National Institute on Aging-Alzheimer's Association guidelines for the neuropathologic assessment of Alzheimer's disease. Alzheimers Dement 2012;58: $1-13$.

8. Mirra SS, Heyman AT, Mckeel D, et al. The consortium to establish a registry for Alzheimer disease (CERAD). Part II. Standardization of the neuropathologic assessment of Alzheimer's disease. Neurology 1991;41:479-486.

9. Thal DR, Rub U, Braak H. Phases of Abeta deposition in the human brain and its relevance for the development of AD. Neurology 2002;58:1791-1800.

10. Mackenzie IRA, Neumann M, Bigio EH, et al. Nomenclature and nosology for neuropathologic subtypes of frontotemporal lobar degeneration: an update. Acta Neuropathol 2010;119:1-4.

11. McKeith IG. Consensus guidelines for the clinical and pathologic diagnosis of dementia with Lewy bodies (DLB): report of the Consortium on DLB International Workshop. J Alzheimers Dis 2006; 9(3 suppl):417-423.

12. Rinne JO, Brooks DJ, Rossor MN, et al. 11C-PiB PET assessment of change in fibrillar amyloid-beta load in patients with Alzhei- mer's disease treated with bapineuzumab: a phase 2, doubleblind, placebo-controlled, ascending-dose study. Lancet Neurol 2010;9:363-372.

13. Salloway S, Sperling R, Fox NC, et al. Two phase 3 trials of bapineuzumab in mild-to-moderate Alzheimer's disease. $\mathrm{N}$ Engl J Med 2014;370:322-333.

14. Doody RS, Thomas RG, Farlow M, et al. Phase 3 trials of solanezumab for mild-to-moderate Alzheimer's disease. $\mathrm{N}$ Engl J Med 2014;370:311-332

15. Petrou M, Dwamena B, Foerster B, et al. Amyloid deposition in Parkinson's disease and cognitive impairment: a systematic review. Mov Disord 2015;30:928-935.

16. Serrano-Pozo A, Qian J, Monsell SE, et al. Mild to moderate Alzheimer dementia with insufficient neuropathological changes. Ann Neurol 2014;75:597-601.

17. Beach TG, Monsell SE, Phillips LE, Kukull W. Accuracy of the clinical diagnosis of Alzheimer disease at National Institute on Aging Alzheimer Disease Centers, 2005-2010. J Neuropathol Exp Neurol 2012;71:266-273.

18. Rabinovici GD, Jagust WJ, Furst AJ, et al. Abeta amyloid and glucose metabolism in three variants of primary progressive aphasia. Ann Neurol 2008;64:388-401.

19. Kantarci K, Lowe VJ, Boeve BF, et al. Multimodality imaging characteristics of dementia with Lewy bodies. Neurobiol Aging 2012; 33:2091-2105.

20. Karageorgiou E, Miller BL. Frontotemporal lobar dementia: a clinical approach. Sem Neurol 2014;34:189-201.

21. Maruyama $M$, Shimada $H$, Suhara $T$, et al. Imaging of tau pathology in a tauopathy mouse model and in Alzheimer patients compared to normal controls. Neuron 2013;79:1094-1108.

22. Dugger BN, Clark CM, Serrano G, et al. Neuropathologic heterogeneity does not impair florbetapir-positron emission tomography postmortem correlates. J Neuropathol Exp Neurol 2014;73:72-80.

23. Clark CM, Pontecorvo MJ, Beach TG, et al. Cerebral PET with florbetapir compared with neuropathology at autopsy for detection of neuritic amyloid- $\beta$ plaques: a prospective cohort study. Lancet Neurol 2012;11:669-678.

24. Clark CM, Schneider JA, Bedell BJ, et al. Use of florbetapir-PET for imaging beta-amyloid pathology. JAMA 2011;305:275-283.

25. Curtis C, Gamez JE, Singh $U$, et al. Phase 3 trial of flutemetamol labeled with radioactive fluorine 18 imaging and neuritic plaque density. JAMA Neurol 2015;72:287-294.

26. Murray ME, Lowe VJ, Graff-Radford NR, et al. Clinicopathologic and 11C-pittsburgh compound B implications of Thal amyloid phase across the Alzheimer's disease spectrum. Brain 2015;138: 1370-1381.

27. Albin RL, Koeppe RA, Burke JF, et al. Comparing fludeoxyglucose F18-PET assessment of regional cerebral glucose metabolism and [11C]dihydrotetrabenazine-PET in evaluation of early dementia and mild cognitive impairment. Arch Neurol 2010;67:440-446.

28. Koeppe RA, Gilman S, Joshi A, et al. 11C-DTBZ and 18F-FDG PET measures in differentiating dementias. J Nucl Med 2005;46: 936-944 